\title{
Microsurgical anatomy of the inferior limiting insular sulcus and the temporal stem
}

\author{
Eduardo Carvalhal Ribas, MD, ${ }^{1,2}$ Kaan Yagmurlu, MD, ${ }^{1}$ Hung Tzu Wen, MD, ${ }^{2}$ and \\ Albert L. Rhoton Jr., MD' \\ 1Department of Neurosurgery, University of Florida, Gainesville, Florida; and 2Division of Neurosurgery, Hospital das Clínicas, \\ University of São Paulo Medical School, São Paulo, Brazil
}

\begin{abstract}
OBJECT The purpose of this study was to describe the location of each white matter pathway in the area between the inferior limiting insular sulcus (ILS) and temporal horn that may be crossed in approaches through the temporal stem to the medial temporal lobe.
\end{abstract}
METHODS The fiber tracts in 14 adult cadaveric cerebral hemispheres were examined using the Klingler technique. The fiber dissections were completed in a stepwise manner, identifying each white matter pathway in different planes and describing its position in relation to the anterior end of the ILS.

RESULTS The short-association fibers from the extreme capsule, which continue toward the operculae, are the most superficial subcortical layer deep to the ILS. The external capsule fibers are found deeper at an intermediate layer and are formed by the uncinate fasciculus, inferior frontooccipital fasciculus, and claustrocortical fibers in a sequential anteroposterior disposition. The anterior commissure forms the next deeper layer, and the optic radiations in the sublenticular part of the internal capsule represent the deepest layer. The uncinate fasciculus is found deep to the anterior third of the ILS, whereas the inferior frontooccipital fasciculus and optic radiations are found superficial and deep, respectively, at the posterior two-thirds of this length.

CONCLUSIONS The authors' findings suggest that in the transsylvian approach, a 6-mm incision beginning just posterior to the limen insula through the ILS will cross the uncinate fasciculus but not the inferior frontooccipital fasciculus or optic radiations, but that longer incisions carry a risk to language and visual functions.

http://thejns.org/doi/abs/10.3171/2014.10.JNS141194

KEY WORDS white matter fibers; inferior limiting insular sulcus; temporal stem; microsurgical anatomy; cerebrum; transsylvian approach

$\mathrm{T}$ HE temporal stem is a widely used term, but is still a controversial concept. Definitions of the stem range from its being as small as the fiber bundles passing between the limen insula and the anterior end of the temporal horn, to its being as large as including all the fibers passing beneath the length of the inferior limiting insular sulcus (ILS). ${ }^{13,42,45,46}$ The transsylvian approach to the medial temporal lobe is commonly directed through or adjacent to the ILS and temporal stem, where opening of the temporal horn and exposure of the amygdala and the hippocampus are key steps. Unfortunately, the temporal lobe is the site of important brain functions, and complications involving visual and language deficits are not rare..$^{12,34,38}$
In this study, based on the larger definition of the stem, the location of each white matter pathway between the ILS and temporal horn was described, with special attention to their relation to the limen insula. The meeting point of the ILS with the limen insula can be identified during surgery and used as a landmark to estimate the position of each set of fibers beneath the ILS.

\section{Methods}

Fourteen adult cadaveric cerebral hemispheres were examined using $\times 3$ to $\times 40$ magnification. The specimens were fixed with formalin and preserved in a $70 \%$ alcohol

ABBREVIATIONS DTI = diffusion tensor imaging; ILS = inferior limiting insular sulcus; LGB = lateral geniculate body; TLP = temporal limen point.

SUBMITTED May 29, 2014. ACCEPTED October 8, 2014.

INCLUDE WHEN CITING Published online April 10, 2015; DOI: 10.3171/2014.10.JNS141194.

DISCLOSURE This research was funded by the University of Florida Foundation. The authors report no conflict of interest concerning the materials or methods used in this study or the findings specified in this paper. 
solution. The vessels were perfused with colored latex, and fiber dissection was performed after freezing, a technique first described by Klingler. ${ }^{23}$ This technique allows identification of the main fiber tracts and has been shown to have a good correlation with other methods of fiber identification, such as diffusion tensor imaging (DTI). ${ }^{26,35,43}$

The dissection was done in a stepwise manner. The position of the white matter pathways found in each deeper step of these dissections was noted according to their distance from the anterior and posterior limit of the ILS. The anterior limit was regarded as the point where the limen insula joins the ILS, named here the temporal limen point (TLP), as opposed to the frontal limen point, where the limen insula joins the anterior limiting sulcus. The posterior limit was established as the point where the ILS joins the superior limiting sulcus, named the posterior insular point. ${ }^{42}$ The TLP was chosen as an important landmark because it is easily found during surgery after splitting the sylvian fissure. All measurements were made independently by the first and second authors, and the final values are the average of both findings (Figs. 1 and 2; Table 1).

Fine straight needles were placed along the calcarine fissure, perpendicular to its mesial cortical surface, to identify the location of this fissure in relation to the lateral surface of the brain. This made it possible to distinguish the portion of the visual radiation directed to the superior and inferior lips of the calcarine fissure.

Measurements were made with an electric digital caliper, and SPSS software (IBM, Inc.) was used to calculate the SD for each set of measurements (Table 1). Digital single-lens reflex (SLR) cameras (Nikon and Cannon) were used for photographic documentation, and the pictures were processed by High-Dynamic-Range Imaging (HDRI or HDR) with the software Photomatix Pro (HDRsoft). This method can combine several pictures taken from the same point of view, but with different exposure values, enhancing the contrast of the final picture to better display the white matter pathways.

\section{Results \\ Cortical Layer}

The insula lies at the depth of the sylvian fissure, covered by opercula of the frontal, parietal, and temporal lobes (Figs. 1 and 3). ${ }^{40,42}$ It has a triangular shape, with its vertex pointing anterior and inferior to the limen insula. It is separated from the opercula by a shallow sulcus, called the limiting or circular sulcus of the insula. The limiting sulcus is subdivided into 3 parts: inferior, anterior, and superior. The ILS extends from the posterior insular point where this sulcus meets the superior limiting sulcus to the point where the ILS joins the limen insula, named here the TLP (Fig. 1). The anterior limiting sulcus, located between the orbitofrontal operculum and the anterior surface of the insula, joins the limen insula at a point called the frontal limen point. The superior limiting sulcus is located medial to the frontoparietal operculum.

The temporal operculum, as it faces the inferior part of the insular surface, has to be retracted to expose the ILS. Its lateral surface is represented by the superior temporal gyrus, and its medial surface is divided into an ante- rior part called the polar plane, which is composed of the small "sulci and gyri of Schwalbe," and a posterior part called the temporal plane, which is formed by the transverse temporal gyri. ${ }^{40,42}$ The most anterior transverse temporal gyrus, named Heschl's gyrus, is directed posteriorly and medially toward the posterior insular point.

The ILS is curved and can be subdivided into an anterior and posterior part (Fig. 1D and E). The anterior part is medial to the polar plane and has a horizontal orientation, whereas the posterior part runs along the medial margin of Heschl's gyrus and assumes a more vertical orientation. The point that subdivides the ILS, named the posteroinferior insular point, lies medial to the anterior part of Heschl's gyrus and lateral to the deep posterior part of the lateral geniculate body (LGB). ${ }^{45}$ This point is particularly important for locating the optic radiations, since they originate from the LGB and progress toward the temporal and parietal lobes before ending at the occipital lobe. The length of the ILS averaged $43.7 \mathrm{~mm}$ (see Table 1 for range and SD). The length of the anterior part of the ILS, from the TLP to the LGB, averaged $29.2 \mathrm{~mm}$, and the posterior part, from the LGB to the posterior insular point, averaged $14.5 \mathrm{~mm}$. Similar findings have been reported by Wang et al. ${ }^{45}$ (Fig. 2; Table 1).

\section{Superficial White Matter Layer-Extreme Capsule}

The most superficial white matter layer, the extreme capsule, is found directly under the thin cortical mantle on the insular surface. These fibers have a centrifugal direction, spreading from the insular gyri to the mesial surface of all the opercula. They course along the external surface of the claustrum at the same depth as the short-association fibers that interconnect the insular gyri (Figs. 3 and 4).

The claustrum is a thin collection of gray matter that has a ventral and a dorsal portion (Fig. 3C). The ventral portion lies deep to the anteroinferior part of the insular surface and its gray matter is poorly organized, resembling small islands embedded into white matter pathways. The dorsal portion of the claustrum is more posterior and superior, and its gray matter is more densely organized, assuming the shape of a thin dark sheet. The most inferior limit of the claustrum continues under the anterior part of the ILS toward the amygdala, and was not seen under the posterior part of the ILS.

\section{Intermediate White Matter Layer-External Capsule and Anterior Commissure}

The external capsule, found between the claustrum and the putamen, is also a group of fibers that passes under the limiting sulci to reach the cerebrum. The external capsule is composed of 3 fiber pathways, all located at the same depth: the uncinate and inferior frontooccipital fasciculi, and the claustrocortical fibers (Figs. 3-5).

The uncinate fasciculus is the most anterior component of the external capsule (Figs. 3C and 4). This longassociation fiber bundle originates at the frontoorbital and inferolateral frontal lobe, and it passes under the anterior limiting sulcus to reach the anteroinferior part of the insula, where it makes a sharp turn inferiorly and anteriorly to pass under the anterior part of the ILS and continue 

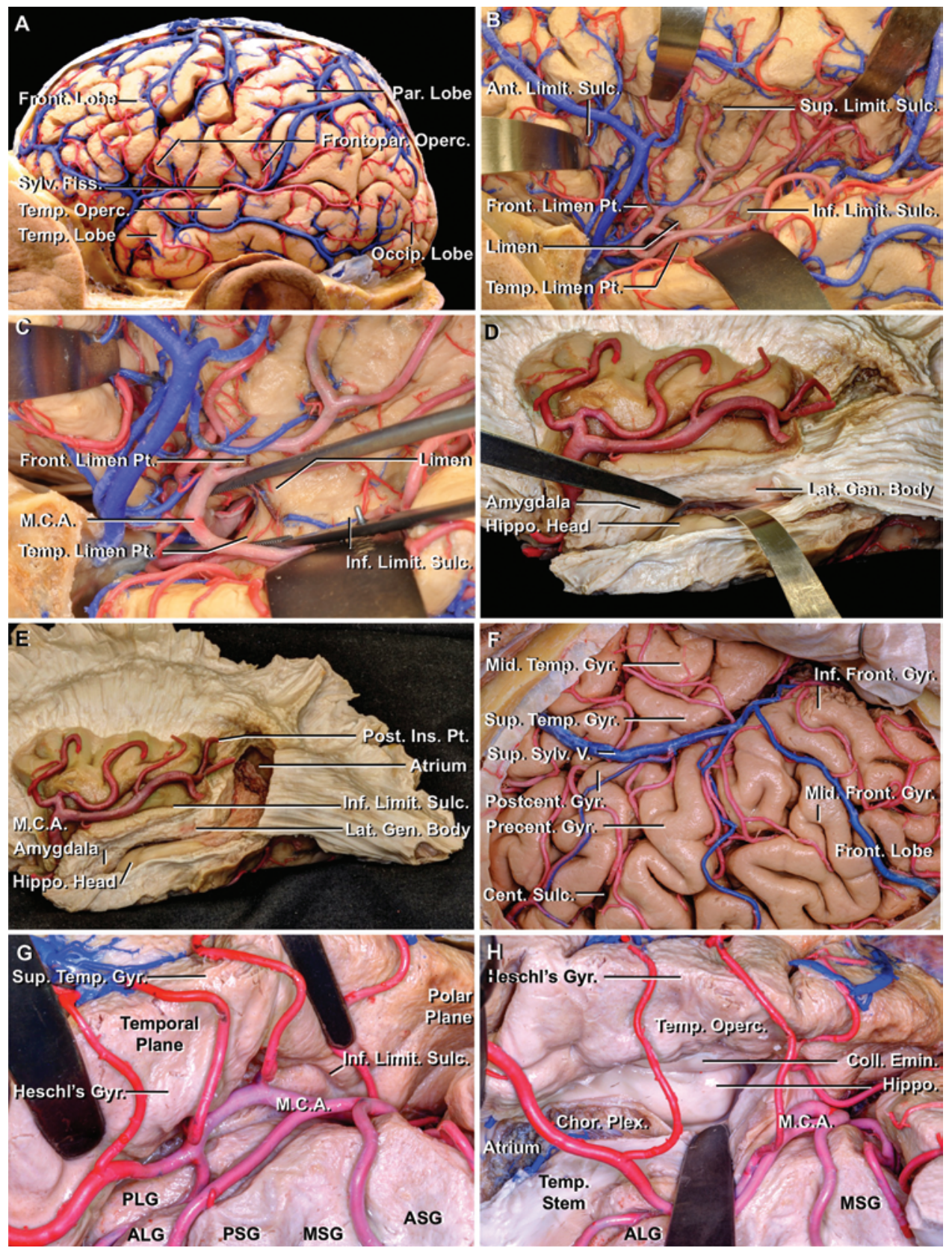

FIG. 1. A: The frontal, parietal, and temporal opercula border the sylvian fissure and overlap and hide the insula. B: The sylvian fissure has been opened. The insula is bounded anteriorly by the anterior insular sulcus, covered by the orbitofrontal operculum; superiorly by the superior insular sulcus, covered by the frontoparietal operculum; and inferiorly by the ILS, covered by the temporal operculum. The limen insula, found at the anteroinferior vertex of the insula, crosses the anterior limiting sulcus at the frontal limen point and the ILS at the TLP. C: Enlarged view showing the junction of the limen insula with the anterior and inferior limiting sulci at the frontal and temporal limen points, respectively. The middle cerebral artery (MCA) bifurcates near the limen insula. D: The cortex, except that covering the insula, has been removed to the depth of the retro- and sublenticular portion of the internal capsule. The anterior part of the ILS has been opened to expose the temporal horn. The amygdala, located directly under the TLP, has an anterior extraventricular portion and a posterior intraventricular portion. The head of the hippocampus is positioned in the floor of the temporal horn below and behind the amygdala. All the fiber pathways between the TLP and the LGB have been transected, but the optic radiations directed to the superior lip of the calcarine fissure are spared. E: The incision at the ILS has been extended to the posterior insular point. The ILS is curved and can be divided at a flexion point located lateral to the LGB. F: A left frontotemporal craniotomy has been completed to expose the sylvian fissure. G: The temporal operculum has been retracted to expose the insular cortex and MCA. The ILS is incised to transect the temporal stem and to access the temporal horn for removal of the medial temporal structures. H: The ILS has been opened as far posteriorly as the atrium, and the choroid plexus, hippocampus, and the collateral eminence in the temporal horn have been exposed. ALG = anterior long gyrus; Ant. = anterior; ASG = anterior short gyrus; Cent. = central; Chor. Plex. = choroid plexus; Coll. Emin. = collateral eminence; Fiss. = fissure; Front. = frontal; Frontopar. = frontoparietal; Gen. = geniculate; Gyr. = gyrus; Hippo. = hippocampus/hippocampal; Inf. = inferior; Ins. = insular; Lat. = lateral; Limit. = limiting; Mid. = middle; MSG = middle short gyrus; Occip. = occipital; Operc. $=$ operculum; Par. = parietal; $P L G=$ posterior long gyrus; Post. = posterior; Postcent. = postcentral; Precent. = precentral; $P S G=$ posterior short gyrus; Pt. = point; Sulc. $=$ sulcus; Sup. = superior; Sylv. = sylvian; Temp. $=$ temporal; V. $=$ vein . 


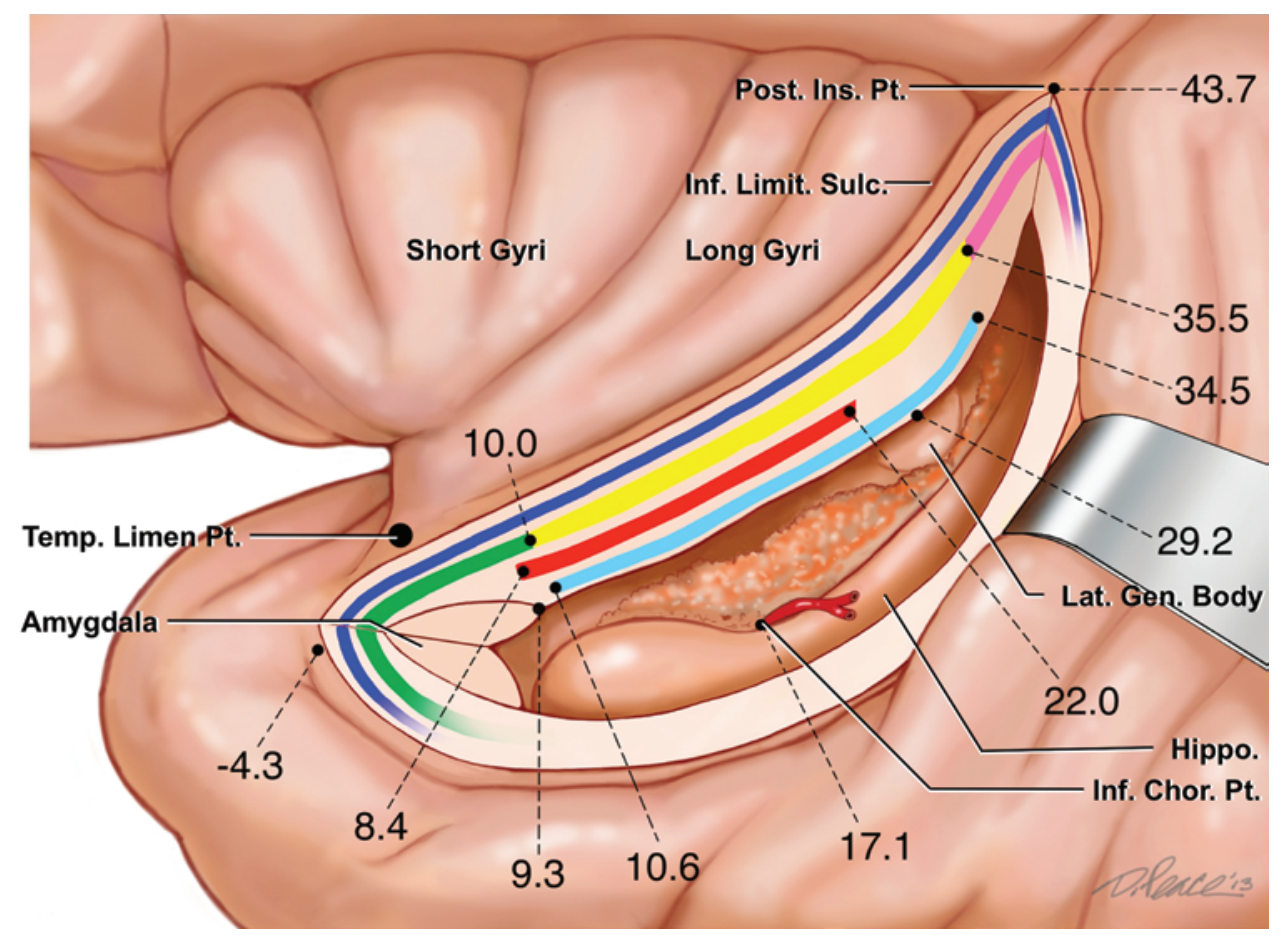

FIG. 2. Schematic representation of the location of the fiber tracts in the temporal stem. The temporal stem extends along the lower margin of the insula adjacent to the long gyri and ILS. The average distance in millimeters between the TLP and the anterior and posterior edge of each tract is shown in this figure and in Table 1, which also shows the range and SD of measurements. The most superficial fibers in the temporal stem are the short-association fibers from the extreme capsule (deep blue) that continue toward the operculum. The external capsular fibers found deeper at an intermediate level are formed by the uncinate fasciculus (green), the inferior frontooccipital fasciculus (yellow), and the claustrocortical fibers (pink) sequentially in an anterior to posterior direction. The next deeper fibers are those crossing in the anterior commissure (red). The deepest layers of fibers in the temporal stem are the optic radiations (light blue). Their distance from the limen insula at the anterior end of the ILS averaged as follows: uncinate fasciculus posterior limit at junction with inferior frontooccipital fasciculus anterior limit, $10 \mathrm{~mm}$; inferior frontooccipital fasciculus posterior limit at junction with claustrocortical fibers anterior limit, $35.5 \mathrm{~mm}$. The anterior commissure lies deeper, and its anterior and posterior limits averaged 8.4 and $22 \mathrm{~mm}$, respectively, posterior to the limen insula. The optic radiations, part of the sublenticular part of the internal capsule, represent the deeper layer and were found between an average of 10.6 and 34.5 $\mathrm{mm}$ posterior to the limen insula. The LGB was located an average of $29.2 \mathrm{~mm}$ posterior to the limen. Chor. = choroidal; Gen. = geniculate; Hippo. = hippocampus; Inf. = inferior; Ins. = insular; Lat. = lateral; Limit. = limiting; Post. = posterior; Pt. = point; Sulc. = sulcus; Temp. $=$ temporal. Copyright Albert $\mathrm{L}$. Rhoton Jr. Published with permission.

to the temporal pole. This curve results in the uncinate fasciculus' hook-like appearance around the limen insula.

The inferior frontooccipital fasciculus, also a longassociation fiber bundle, is located directly superior and posterior to the uncinate fasciculus (Figs. 3D, 4, and 5B). It originates at the inferior frontal gyrus, passes under the anterior limiting sulcus, occupies the anterior part of the insula just superior to the uncinate fasciculus, and continues posteriorly, passing under the ILS toward the posterior part of the temporal, parietal, and occipital lobes.

The uncinate fasciculus and the inferior frontooccipital fasciculus are located deep to the anteroinferior part of the insular surface, and both pass through the ventral claustrum. As these fibers intermingle with the islands of gray matter of the ventral claustrum, they contribute to both the extreme and external capsule in this region. ${ }^{17}$ The dorsal claustrum projects to the frontal and parietal lobes. These claustrocortical fibers have a radial configuration centered at the dorsal claustrum, projecting toward the frontal and parietal lobes, and passing under the limiting sulci.

Deep to the ILS, there is no natural separation be- tween the uncinate and inferior frontooccipital fasciculi. However, since the uncinate fasciculus is directed anteriorly to the temporal pole and the inferior frontooccipital fasciculus progresses posteriorly, the transition point between them was regarded as the point where the fibers assume a perpendicular disposition in relation to the ILS. The transition point between the inferior frontooccipital fasciculus and the claustrocortical fibers is more clearly seen, because the fibers from the inferior frontooccipital fasciculus can be followed from the frontal to the occipital lobe, whereas the claustrocortical fibers arise from the dorsal claustrum.

The course of the uncinate fasciculus under the ILS begins at the TLP level and continues to the uncinate/inferior frontooccipital fasciculi transition point located an average of $10 \mathrm{~mm}$ posterior to the TLP (Fig. 2; Table 1). This distance corresponds to the width of the uncinate fasciculus under the ILS. The inferior frontooccipital fasciculus under the ILS was found between the uncinate/inferior frontooccipital fasciculi transition point and the inferior frontooccipital fasciculus/claustrocortical fibers transition 
TABLE 1. Measurements of the ILS ( $\mathrm{mm})$ in 14 cadaveric hemispheres

\begin{tabular}{lcc}
\hline \multicolumn{1}{c}{ ILS } & Average (range) & SD \\
\hline Total length & $43.7(38.5-50.1)$ & \pm 3.6 \\
\hline Anterior part (TLP to LGB) & $29.2(22.4-37.0)$ & \pm 4.0 \\
\hline Posterior part (LGB to posterior insular point) & $14.5(8.2-20.3)$ & \pm 3.4 \\
\hline Distance along ILS from the TLP to & $0.0(0.0-0.0)$ & \pm 0.0 \\
\hline 1. Uncinate fasciculus, anterior limit & $10.0(6.4-14.5)$ & \pm 2.2 \\
\hline 2. Uncinate fasciculus, posterior limit (junction w/ inferior frontooccipital fasciculus) & $35.5(29.2-40.0)$ & \pm 2.7 \\
\hline 3. Junction of inferior frontooccipital fasciculus w/ claustrocortical fibers & $8.4(3.2-10.2)$ & \pm 1.8 \\
\hline 4. Anterior commissure, anterior limit & $22.0(12.1-34.6)$ & \pm 6.8 \\
\hline 5. Anterior commissure, posterior limit & $10.6(6.0-17.7)$ & \pm 3.4 \\
\hline 6. Optic radiations, anterior limit & $34.5(28.7-39.1)$ & \pm 3.5 \\
\hline 7. Optic radiations, posterior limit & $-4.3(-7.1$ to -2.0) & \pm 1.5 \\
\hline 8. Amygdala, anterior limit (anterior to TLP) & $9.3(5.5-14.4)$ & \pm 2.6 \\
\hline 9. Amygdala, posterior limit & $4.4(0.0-9.5)$ & \pm 2.9 \\
\hline 10. Temporal horn, anterior limit & $17.1(12.6-24.1)$ & \pm 3.8 \\
\hline 11. Inferior choroidal point & & \\
\hline Length of each structure under the ILS & $10.0(6.4-14.5)$ & \pm 2.2 \\
\hline Uncinate fasciculus & $25.5(21.1-30.1)$ & \pm 2.6 \\
\hline Inferior frontooccipital fasciculus & $13.7(6.3-26.4)$ & \pm 6.7 \\
\hline Anterior commissure & $23.9(18.9-32.5)$ & \pm 3.6 \\
\hline Optic radiations & $13.6(8.2-21.5)$ & \pm 3.8 \\
\hline Amygdala & & \\
\hline
\end{tabular}

point. This second point that marks the beginning of the claustrocortical fibers was located at an average of 35.5 $\mathrm{mm}$ posterior to the TLP. The width of the inferior frontooccipital fasciculus under the ILS averaged $25.5 \mathrm{~mm}$. The claustrocortical fibers were found along the entire length of the ILS posterior to the inferior frontooccipital fasciculus/claustrocortical fibers transition point, and also under the superior limiting sulcus (Figs. 2, 3C and D, 4, and 5).

The anterior commissure belongs to the commissural fiber system of the brain, interconnecting both hemispheres. Its medial component has a cylinder or "handlebar" appearance that crosses the midline between the pre- and postcommissural fibers of the fornix (Figs. 3F, $4 \mathrm{~B}$, and $5 \mathrm{C}$ ). It has a ventral extension to the septal region, interconnecting olfactory structures, and a lateral extension, which continues to the temporal lobe. This lateral extension passes through a glial tunnel at the anteroinferior pole of the globus pallidus, named "Gratiolet's canal," and more laterally under the ILS. The fibers of the anterior commissure are positioned in a plane deep to the uncinate and inferior frontooccipital fasciculi, and at the temporal lobe they can be followed to the temporal pole and middle and inferior temporal gyri. The lateral extension of the anterior commissure under the ILS was found between an average of $8.4 \mathrm{~mm}$ and $22 \mathrm{~mm}$ posterior to the TLP. Its width under the ILS averaged $13.7 \mathrm{~mm}$ (Fig. 2; Table 1).

\section{Deep White Matter Layer-Visual and Auditory Radiations}

As the dissection progresses deeper under the external capsule, the putamen is exposed, followed by the globus pallidus. These 2 collections of gray matter are located deep to the insular surface, but not below the trajectory from the ILS to the temporal horn. Their removal exposes the internal capsule.

The internal capsule is a thick group of fibers that occupies the space between the lentiform nucleus laterally, and the caudate nucleus medially. It includes most of the projection system of the cerebrum, and more importantly, the pyramidal corticopontocerebellar tracts and all thalamic radiations. The internal capsule is traditionally divided into an anterior limb, genu, posterior limb, and retrolenticular and sublenticular portions. The retrolenticular and sublenticular portions of the internal capsule pass below the posterior and anterior parts of the ILS, respectively.

The retrolenticular portion of the internal capsule, named for the fact that these fibers are posterior to the lentiform nucleus, connects the caudal portions of the thalamus, including the pulvinar and LGB, with the posterior parietal gyri and the occipital lobe. The fibers that arise from the LGB and project to the lips of the calcarine fissure carry visual inputs, pass under the ILS, and are traditionally divided into anterior, central, and posterior bundles. ${ }^{12}$ The sublenticular part of the internal capsule is small and interconnects the posterior thalamus and the medial geniculate body to the temporal cortex and transverse temporal gyri. Among these fibers are the auditory radiations, which course from the medial geniculate body to the anterior transverse temporal (Heschl's) gyrus.

As the basal ganglia are removed in a lateral-to-medial direction, the optic radiation can be identified as originating at the LGB and progressing toward the calcarine fissure (Figs. 3G, 4C and D, and 5D). Although classically divided into anterior, central, and posterior parts, the optic 

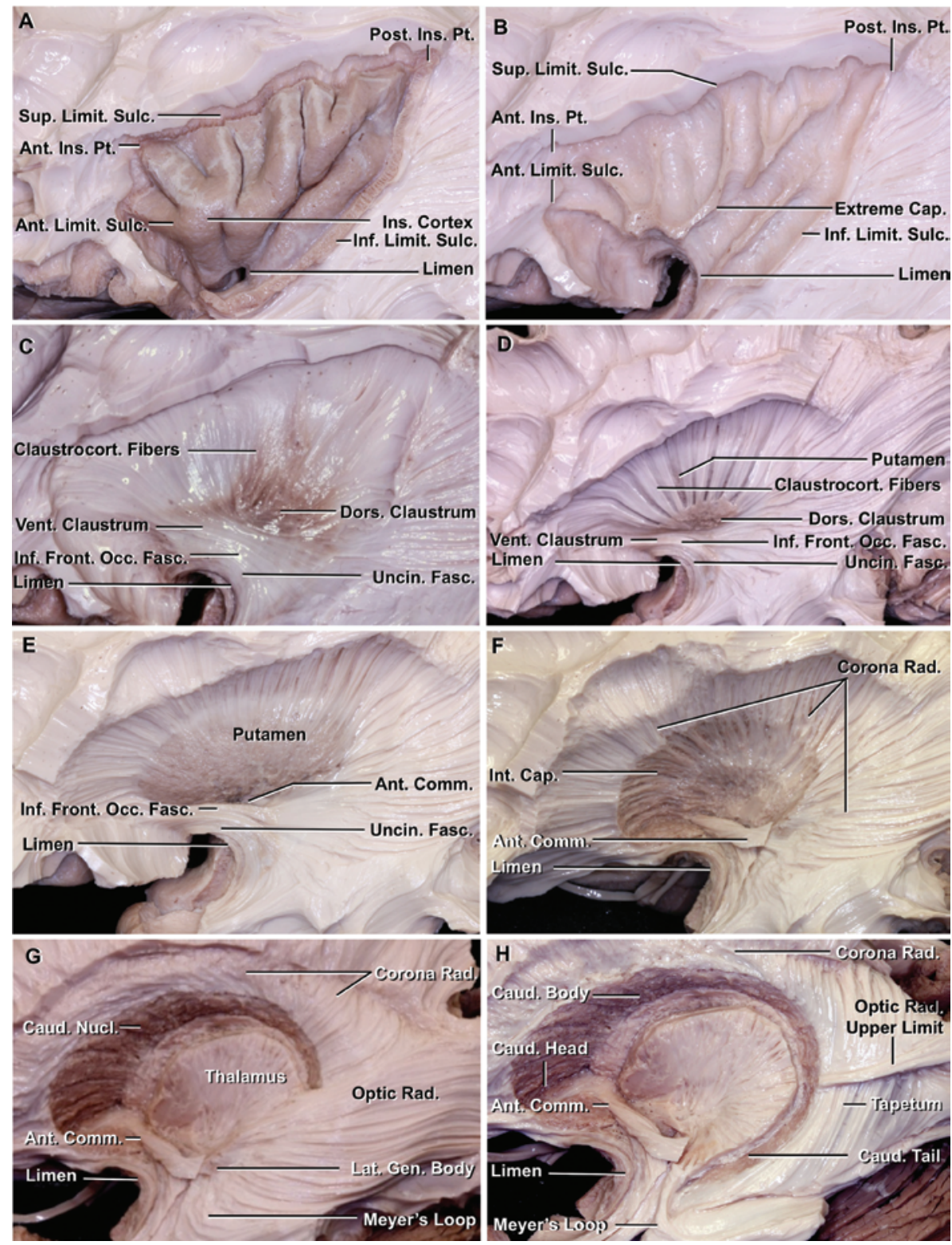

FIG. 3. Stepwise insular dissection. A: The opercula were removed to expose the right insular cortex. The insula is bounded anteriorly by the anterior limiting sulcus, superiorly by the superior limiting sulcus, and inferiorly by the ILS. The limen insula is found at its anteroinferior vertex. The meeting point of the anterior and superior limiting sulci is named the anterior insular point and the meeting point of the superior and inferior limiting sulci is called the posterior insular point. B: The insular cortex was removed to expose the extreme capsule, which is composed of short-association fibers that interconnect the insular gyri and spread beneath the limiting sulci toward the opercula. C: Removing the extreme capsule exposes the claustrum. The dorsal portion of the claustrum is more densely organized, forming a thin gray matter sheet, from which the claustrocortical fibers arise and radiate under the superior and inferior limiting sulci. The ventral claustrum is loosely organized into small islands of gray matter sprinkled in the uncinate fasciculus and the inferior frontooccipital fasciculus that give a brownish color to the region. The uncinate fasciculus, inferior frontooccipital fasciculus, and claustrocortical fibers all course at the same depth under the ILS and can be seen in sequence posterior to the limen insula. D: Small windows were opened in the claustrocortical fibers to expose the putamen. E: The claustrum and claustrocortical fibers were removed to expose the putamen. The anterior commissure can be seen at the anteroinferior pole of the putamen in Gratiolet's canal, from which it progresses laterally to pass under the ILS and spreads to the temporal and occipital lobes. F: The putamen, globus pallidus, uncinate fasciculus, and inferior frontooccipital fasciculus were removed. The internal capsule lies medial to the putamen and globus pallidus and lateral to the thalamus. These fibers of the internal capsule project to the cerebral convexity. G: The internal capsule has been removed. The optic radiations arise from the LGB, located at the posteroinferior aspect of the thalamus. The most anterior portion of the optic radiations, called Meyer's loop, progresses anteriorly toward the temporal pole before turning inferior and posterior to terminate at the inferior lip of the calcarine fissure. The fibers that arise from the lateral and posterior aspect of the LGB have a more direct course to the occipital pole. The fibers arising from the superior aspect of the LGB are directed to the superior lip of the calcarine fissure. H: All the optic radiations, except the anterior edge of Meyer's loop and the upper edge lateral to the atrium, have been removed to expose the tapetal fibers forming the roof and lateral wall of the temporal horn. The caudate tail has been exposed in the roof of the temporal horn. Ant. = anterior; Cap. = capsule; Caud. = caudate; Claustrocort. = claustrocortical; Comm. = commissure; Dors. = dorsal; Fasc. = fasciculus; Front. = frontal; Gen. = geniculate; Inf. = inferior; Ins. = insular; Int. = interior; Lat. = lateral; Limit. = limiting; Nucl. = nucleus; Occ. = occipital; Post. = posterior; Pt. = point; Rad. = radiata/radiations; Sulc. = sulcus; Sup. = superior; Uncin. = uncinate; Vent. = ventral. 

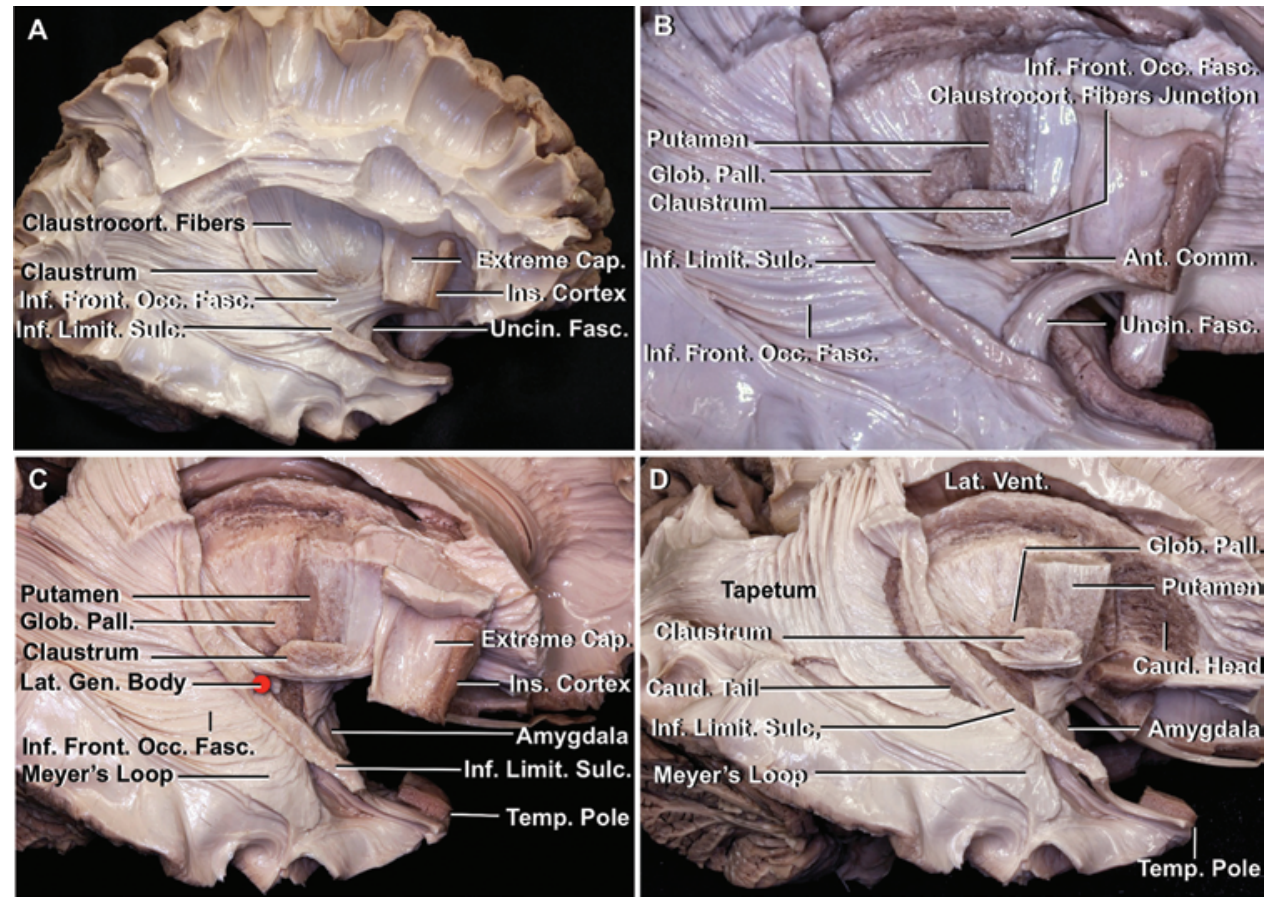

FIG. 4. The fibers deep to the right insular cortex and ILS travel at different depths, but are presented together in a stepwise dissection to highlight the relationships among them. A: The extreme capsule found beneath the insular cortex covers the entire insula. The external capsule is composed of the uncinate fasciculus, inferior frontooccipital fasciculus, and claustrocortical fibers, which course at the same depth deep to the claustrum. However, some of these fibers also pass superficial to the claustrum. The uncinate fasciculus passes deep to the limen insula. The inferior frontooccipital fasciculus is located posterior to the uncinate fasciculus and both pass under the anterior part of the ILS. The claustrocortical fibers arise in the dorsal claustrum and pass under the posterior portion of the ILS and the superior limiting sulcus in their course toward the frontal and parietal lobes. B: Some claustrocortical fibers have been removed to expose the putamen medially. The globus pallidus is located deep to the putamen. Some of the inferior frontooccipital fasciculus fibers at the anterior part of the insula were removed to expose the anterior commissure that will progress laterally to the temporal and occipital lobes at a deeper level. C: The part of the uncinate fasciculus and inferior frontooccipital fasciculus under the anterior part of the ILS and insula has been removed to expose the amygdala and optic radiations. The amygdala is located at the anterior tip of the temporal horn and blends into the lower part of the putamen without a clear demarcation between the two nuclei. The optic radiations arise from the LGB, located at the red pin, and terminate along the calcarine fissure. Meyer's loop, the anterior bundle of the optic radiations, has an initial anterior course before turning posteriorly toward the occipital lobe. D: The extreme capsule, insular cortex, and anterior third of the putamen have been removed to expose the head and tail of the caudate nucleus, some of the optic radiations, and tapetum. Ant. = anterior; Cap. = capsule; Caud. = caudate; Claustrocort. = claustrocortical; Comm. = commissure; Fasc. = fasciculus; Front. = frontal; Gen. = geniculate; Glob. Pall. = globus pallidus; Inf. = inferior; Ins. = insular; Lat. = lateral; Limit. = limiting; Occ. = occipital; Sulc. = sulcus; Temp. = temporal; Uncin. = uncinate; Vent. = ventricle.

radiation fibers constitute a continuum of fibers that cover the superior and lateral surfaces of the temporal horn and the atrium. They pass above and below the occipital horn to reach the superior (cuneal) and the inferior (lingual) lips, respectively, of the posterior half of the calcarine fissure. The more anterior fibers arising at the inferior aspect of the LGB (anterior bundle) assume first an anterior course toward the temporal pole (Meyer's loop), and later curve posteriorly to finish at the inferior lip of the calcarine fissure; ${ }^{29}$ the fibers arising at the posterior aspect of the LGB have a more direct posterior course to end at the occipital pole (central bundle); and the fibers arising from the superior aspect of the LGB project to the superior lip of the calcarine fissure (posterior bundle). The anterior and posterior limits of the optic radiations were found under the ILS at an average of $10.6 \mathrm{~mm}$ and $34.5 \mathrm{~mm}$ posterior to the TLP, respectively. The width of the optic radiations under the ILS averaged $23.9 \mathrm{~mm}$ (Fig. 2; Table 1).

\section{Amygdala, Ependyma, and Temporal Horn of the Lateral Ventricle}

Opening the ependymal layer lining the ventricle exposes and provides access to the structures bordering the temporal horn (Fig. 1H). The amygdala was found directly below the TLP in all cases. The anterior limit of the amygdala was located an average of $4.3 \mathrm{~mm}$ anterior to the TLP, and its posterior limit was located at an average of $9.3 \mathrm{~mm}$ posterior to the TLP. The length of the amygdala under the ILS averaged $13.6 \mathrm{~mm}$. The location of the anterior limit of the temporal horn under the ILS was found at an average of $4.4 \mathrm{~mm}$ posterior to the TLP. Despite the significant variations in these findings, which are probably related to the usual variation of size of the ventricular cavities between different individuals, the amygdala was always found to have an anterior extraventricular and a posterior intraventricular component.

Inside the temporal horn, the amygdala is located an- 


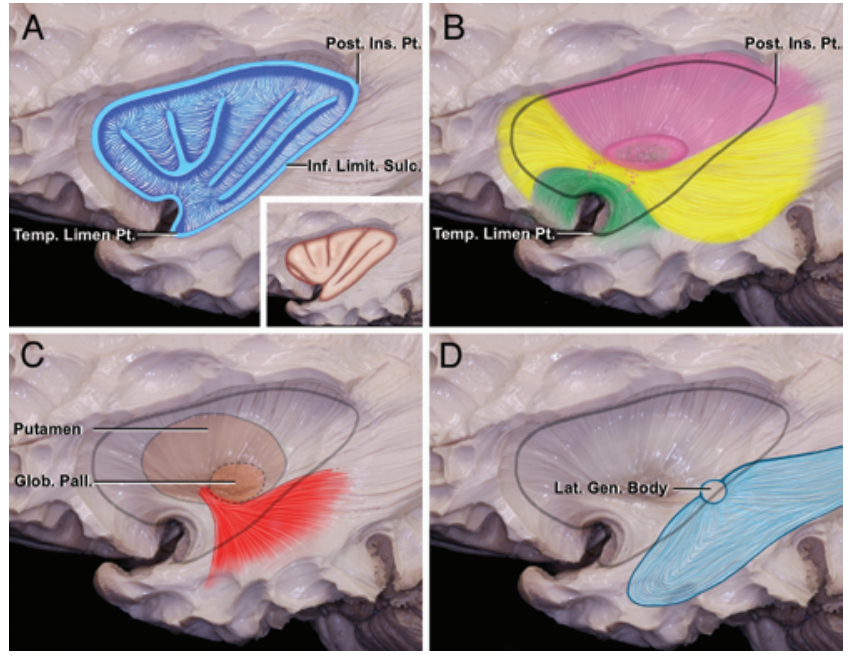

FIG. 5. A-D: Location of fiber tracts in the temporal stem. The temporal stem extends along the lower margin of the insula from the TLP located at the junction of the ILS and limen insula to the posterior insular point positioned at the junction of the superior and inferior limiting sulci. A: The most superficial fibers deep to the ILS course in the extreme capsule formed by the short-association fibers passing from the insula to the operculum. B: The external capsular fibers, found at an intermediate level beneath the ILS, are formed by the uncinate fasciculus (green), the inferior frontooccipital fasciculus (yellow), and claustrocortical fibers (pink). Only a short segment of the claustrocortical fibers pass deep to the ILS. C: The next deeper fibers, crossing below the ILS, are those in the anterior commissure (red). D: The deepest layers of fibers are the optic radiations that cross below the middle third of the ILS (blue). Inf. = inferior; Ins. = insular; Limit. = limiting; Post. = posterior; Pt. = point; Sulc.$=$ sulcus; Temp.$=$ temporal.

terior and superior to the head of the hippocampus (Figs. $1 \mathrm{E}$ and $4 \mathrm{C}$ ). There is a small space that projects medially between the amygdala and hippocampal head called the uncal recess. Immediately posterior to the head of the hippocampus, the anterior choroidal artery exits the crural cistern and enters the temporal horn. This entry point, called the inferior choroidal point, also marks the lower end of the choroidal fissure. ${ }^{31}$ The inferior choroidal point was located below the ILS at an average of $17.1 \mathrm{~mm}$ posterior to the TLP. The transsylvian approach can be combined with the opening of the choroidal fissure to provide access to the contents of the crural and ambiens cisterns. ${ }^{21,30}$

\section{Discussion}

The temporal stem is a widely used term derived from the appearance, on coronal slices of the brain, that the temporal lobe is connected to the basal forebrain underneath the anterior aspect of the insula through a unique peduncle resembling the roots of a tree. ${ }^{22}$ The stem has been defined as being as small as "the portion of white matter that penetrates the temporal lobe between the anterior border of the insula and the inferior horn" and as long as including all fibers extending from the limen insula and the roof of the temporal horn..$^{3,42,45,46}$ This longer definition of the temporal stem includes the sagittal stratum and includes the anterior commissure, uncinate fasciculus, inferior frontooccipital fasciculus, Meyer's loop of the optic radiations, and inferior thalamic fibers. ${ }^{13,23}$ The thickness of the temporal stem, represented by the superoinferior distance between the ILS and the roof of the temporal horn, is reported to average $6.5-8.2 \mathrm{~mm} .{ }^{28,45}$

The temporal horn and mesial temporal lobe structures have been accessed through an anterior temporal lobectomy, the lateral or inferior temporal surface, and more recently by a supracerebellar transtentorial approach., ${ }^{1,4,14,15,20 \text {, }}$ ${ }^{32,36,41}$ However, these approaches have some risk of damaging neocortical structures and white matter pathways or involve an extremely narrow operating field, excessive brain retraction, and potential injury to the vein of Labbé. The transsylvian approach was proposed to reduce these risks. ${ }^{48}$ The approach, as initially described by Wieser and Yaşargil in $1982,{ }^{47}$ included a $15-$ to $20-\mathrm{mm}$ incision at the level of the limen insula and directed toward the temporal horn. Based on the results of this and 2 previous studies from our laboratory, ${ }^{3,37}$ the length of incision described in their 1982 paper would cross part of Meyer's loop. The fact that a group of 173 patients who underwent amygdalohippocampectomy for temporal lobe seizures using this technique contained only 2 patients with visual deficit, both of whom had postoperative hematomas ${ }^{48}$ raised the question of whether our results showing that a 6-mm incision directed backward from the limen would enter Meyer's loop were correct. This was clarified in 2008 by Abdulrauf and Yaşargil in their published comments on a paper on Meyer's loop by Yeni et al. ${ }^{49}$ They acknowledged that the incision in the temporal stem in the 1982 paper by Wieser and Yaşargi ${ }^{47}$ began at the level of the limen insula and moved from "distal to proximal" (not backward, as incorrectly mentioned by Wieser in 1982) in a length of $10-15 \mathrm{~mm}$ over the cortical region of the amygdala, and that the approach was "inaccurately described as transsylvian transinsular by some colleagues," but that it was actually "transsylvian transamygdalar." Thus, the approach described by Wieser and Yaşargil in 1982 did not extend backward but was directed proximally through the amygdala and thus was transsylvian transamygdalar, as advocated in our prior study, and would not be expected to cause a visual loss. ${ }^{3}$

The amygdala is found directly below the TLP, and approaches to the amygdala and hippocampus (e.g., amygdalohippocampectomy) can be made using small incisions directed medially through the amygdala without extension posteriorly in the ILS. After the amygdala is removed, the temporal horn is opened, the head of the hippocampus is exposed, and the inferior choroidal point, where the anterior choroidal artery enters the temporal horn, can be found at an average of $17.1 \mathrm{~mm}$ posterior to the TLP. In their study, Yeni et al. ${ }^{49}$ found that an incision beginning at the limen insula and extending backward $1.5-2 \mathrm{~cm}$ along the ILS resulted in a visual field deficit in $36.6 \%$ of patients. On the other hand, the incision of Wieser and Yaşargil, ${ }^{47}$ as later mentioned, which extended through the cortex covering the amygdala and not along the ILS, was not associated with a visual deficit. The fact that all patients in the series reported by Yeni et al. ${ }^{49}$ did not have a visual deficit seems to imply that the location of Meyer's loop may vary in different individuals, or that part of the anterior bundle can be resected without necessarily causing a major visual deficit.

Based on this and our previous studies,, 37 a 2-cm inci- 
sion extending backward from the limen will, as it deepens, cross some extreme-capsule short-association fibers, the uncinate fasciculus, and the most anterior parts of the inferior frontooccipital fasciculus, anterior commissural fibers, and anterior optic radiations, with a risk to language pathways in the dominant hemisphere and also of homonymous superior quadrantic visual deficit in either hemisphere. ${ }^{3,37}$ Martino et al. ${ }^{28}$ mentioned that the anterior limit of the optic radiations under the ILS was located at the same distance as the uncinate fasciculus/inferior frontooccipital fasciculus transition point, a finding that was also seen in the specimens used for this report.

In moving from superficial to deep along the ILS, the insular cortex has been implicated in a variety of important networks related to olfactory, gustatory, viscerosensory, visceromotor, somatosensory, and vestibular functions, and the primary olfactory cortex and the insular limbic cortex are present at the anterior and intermediate part of the ILS, respectively. ${ }^{33}$ Awake craniotomies have also induced speech arrest with electrical stimulation at the anterior cortex of the insula, but transections of the anterior portion of the ILS have not led to language deficits. ${ }^{8,24,48}$

At a deeper level, an incision extending backward from the TLP first damages the uncinate fasciculus and the inferior frontooccipital fasciculus if carried more posteriorly. Both tracts pass under the ILS, with the transition point between them located an average of $10 \mathrm{~mm}$ posterior to the TLP in our specimens, meaning that an incision smaller than $6 \mathrm{~mm}$ beginning at the TLP would cross the uncinate fasciculus but not damage the inferior frontooccipital fasciculus. Martino et al. ${ }^{28}$ reported similar findings, in which this transition point was located at an average of $10.9 \mathrm{~mm}$ (range $8-15 \mathrm{~mm}$ ) posterior to the limen insula.

Based on subcortical mapping by electrical stimulation during awake neurosurgeries, Duffau et al. ${ }^{6}$ proposed 2 main streams related to language: a ventral and a dorsal one. The ventral stream, represented by the uncinate fasciculus and the inferior frontooccipital fasciculus, is related to the semantic components of language, and is where electrical stimulation induces semantic paraphasias. ${ }^{9,10}$ The dorsal stream is represented by the superior longitudinal fasciculus, where the arcuate fasciculus is its most important subcomponent, and its electrical stimulation has elicited reproducible phonemic paraphasias, namely disorders that affect the phonological form of the words. ${ }^{7}$

A transsylvian-ILS approach will cross part of this ventral semantic stream. The uncinate fasciculus possibly represents a minor component, because its stimulation or resection has not led to language disturbances. ${ }^{9}$ Functional data suggest that the major importance of the inferior frontooccipital fasciculus is in reading, attention, visual processing, and language. ${ }^{2,5,9,10}$ Duffau et al., ${ }^{11}$ based on stimulation and studies of insular gliomas extending into the area of the inferior frontooccipital fasciculus, strongly support it being preserved in the dominant hemisphere. Other studies have supported a role for the uncinate fasciculus in category-specific naming tasks for objects and actions or famous faces. ${ }^{18,25}$ Identifying and preserving the inferior frontooccipital fasciculus also increases the likelihood that the optic radiations on its deep side will be preserved.

The significant variation of the lateral expansion of the anterior commissure suggests that even a small incision at the ILS could damage this set of fibers. ${ }^{33}$ However, there are no reports of deficits related to the anterior commissure in humans.

At a deeper plane, the retrolenticular and sublenticular portions of the internal capsule are found under the ILS. The anterior and posterior limits of the optic radiations were located an average of 10.6 and $34.5 \mathrm{~mm}$ posterior to the TLP, respectively. An incision smaller than $6 \mathrm{~mm}$ beginning at the TLP and extending backward along the ILS would not divide any part of the optic radiations. However, as the incision progresses more posteriorly, the anterior bundle begins to be transected, leading to a risk of causing superior temporal homonymous quadrantanopia. ${ }^{12,38,39,44}$ As the incision approaches the LGB (minimum of 22.4 $\mathrm{mm}$ ), the fibers devoted to the central part of the retina are also put at risk. The sublenticular part also contains the inferior thalamic peduncle including the auditory radiations, temporal corticopontine fiber bundle, and parietooccipital projections.

The DTI technique has the ability to track the main fiber bundles of the brain. There is still no quantitative study reporting the exact location of each fiber pathway at the temporal stem; however, comparative analysis of DTI studies and anatomical dissections have shown a good correlation at this region. ${ }^{16,19,27}$ The DTI technique has the ability to demonstrate several fiber pathways in the same image and possibly better display their relationship, as opposed to fiber dissections, in which some pathways can be displayed only in a stepwise manner. On the other hand, with careful planning, multiple fiber tracts and their relationships can be shown in a single image of a fiber dissection (Fig. 4).

\section{Conclusions}

The cortical and subcortical anatomy of the ILS, with emphasis on the position of each white matter pathway under this sulcus, has been described. Understanding the location of each pathway in the transsylvian approach to the temporal horn is important in preventing damage that could lead to functional deficits.

\section{Acknowledgments}

We deeply appreciate Ms. Robin Barry's assistance with the preparation of the illustrations, Mr. David Peace's contribution of his illustration (Fig. 2), and Ms. Jessica Striley's editorial assistance.

\section{References}

1. Campero A, Tróccoli G, Martins C, Fernandez-Miranda JC, Yasuda A, Rhoton AL Jr: Microsurgical approaches to the medial temporal region: an anatomical study. Neurosurgery 59 (4 Suppl 2):ONS279-ONS308, 2006

2. Catani M, Mesulam M: What is a disconnection syndrome? Cortex 44:911-913, 2008

3. Choi C, Rubino PA, Fernandez-Miranda JC, Abe H, Rhoton AL Jr: Meyer's loop and the optic radiations in the transsylvian approach to the mediobasal temporal lobe. Neurosurgery 59 (4 Suppl 2):ONS228-ONS236, 2006

4. de Oliveira JG, Párraga RG, Chaddad-Neto F, Ribas GC, de Oliveira EP: Supracerebellar transtentorial approachresection of the tentorium instead of an opening-to provide 
broad exposure of the mediobasal temporal lobe: anatomical aspects and surgical applications: clinical article. J Neurosurg 116:764-772, 2012

5. Doricchi F, Thiebaut de Schotten M, Tomaiuolo F, Bartolomeo P: White matter (dis)connections and gray matter (dys) functions in visual neglect: gaining insights into the brain networks of spatial awareness. Cortex 44:983-995, 2008

6. Duffau H: The anatomo-functional connectivity of language revisited. New insights provided by electrostimulation and tractography. Neuropsychologia 46:927-934, 2008

7. Duffau H, Capelle L, Sichez N, Denvil D, Lopes M, Sichez JP, et al: Intraoperative mapping of the subcortical language pathways using direct stimulations. An anatomo-functional study. Brain 125:199-214, 2002

8. Duffau H, Fontaine D: Successful resection of a left insular cavernous angioma using neuronavigation and intraoperative language mapping. Acta Neurochir (Wien) 147:205-208, 2005

9. Duffau H, Gatignol P, Mandonnet E, Capelle L, Taillandier L: Intraoperative subcortical stimulation mapping of language pathways in a consecutive series of 115 patients with Grade II glioma in the left dominant hemisphere. J Neurosurg 109:461-471, 2008

10. Duffau H, Gatignol P, Mandonnet E, Peruzzi P, TzourioMazoyer N, Capelle L: New insights into the anatomofunctional connectivity of the semantic system: a study using cortico-subcortical electrostimulations. Brain 128:797-810, 2005

11. Duffau H, Thiebaut de Schotten M, Mandonnet E: White matter functional connectivity as an additional landmark for dominant temporal lobectomy. J Neurol Neurosurg Psychiatry 79:492-495, 2008

12. Ebeling U, Reulen HJ: Neurosurgical topography of the optic radiation in the temporal lobe. Acta Neurochir (Wien) 92:29-36, 1988

13. Ebeling U, von Cramon D: Topography of the uncinate fascicle and adjacent temporal fiber tracts. Acta Neurochir (Wien) 115:143-148, 1992

14. Falconer MA, Meyer A, Hill D, Mitchell W, Pond DA: Treatment of temporal-lobe epilepsy by temporal lobectomy; a survey of findings and results. Lancet 268:827-835, 1955

15. Fernández-Miranda JC, de Oliveira E, Rubino PA, Wen HT, Rhoton AL Jr: Microvascular anatomy of the medial temporal region: part 1: its application to arteriovenous malformation surgery. Neurosurgery 67 (3 Suppl Operative):ons237ons276, 2010

16. Fernández-Miranda JC, Rhoton AL Jr, Alvarez-Linera J, Kakizawa Y, Choi C, de Oliveira EP: Three-dimensional microsurgical and tractographic anatomy of the white matter of the human brain. Neurosurgery 62 (6 Suppl 3):989-1028, 2008

17. Fernández-Miranda JC, Rhoton AL Jr, Kakizawa Y, Choi C, Alvarez-Linera J: The claustrum and its projection system in the human brain: a microsurgical and tractographic anatomical study. J Neurosurg 108:764-774, 2008

18. Glosser G, Salvucci AE, Chiaravalloti ND: Naming and recognizing famous faces in temporal lobe epilepsy. Neurology 61:81-86, 2003

19. Holl N, Noblet V, Rodrigo S, Dietemann JL, Mekhbi MB, Kehrli P, et al: Temporal lobe association fiber tractography as compared to histology and dissection. Surg Radiol Anat 33:713-722, 2011

20. Hori T, Tabuchi S, Kurosaki M, Kondo S, Takenobu A, Watanabe T: Subtemporal amygdalohippocampectomy for treating medically intractable temporal lobe epilepsy. Neurosurgery 33:50-57, 1993

21. Ikeda K, Shoin K, Mohri M, Kijima T, Someya S, Yamashita J: Surgical indications and microsurgical anatomy of the transchoroidal fissure approach for lesions in and around the ambient cistern. Neurosurgery 50:1114-1120, 2002
22. Kier EL, Staib LH, Davis LM, Bronen RA: MR imaging of the temporal stem: anatomic dissection tractography of the uncinate fasciculus, inferior occipitofrontal fasciculus, and Meyer's loop of the optic radiation. AJNR Am J Neuroradiol 25:677-691, 2004

23. Klingler J: [Development of the macroscopic preparation of the brain through the process of freezing.] Schweiz Arch Neurol Psychiatr 36:247-256, 1935 (Ger)

24. Leal PR, Houtteville JP, Etard O, Emery E: Surgical strategy for insular cavernomas. Acta Neurochir (Wien) 152:16531659,2010

25. Lu LH, Crosson B, Nadeau SE, Heilman KM, GonzalezRothi LJ, Raymer A, et al: Category-specific naming deficits for objects and actions: semantic attribute and grammatical role hypotheses. Neuropsychologia 40:1608-1621, 2002

26. Martino J, da Silva-Freitas R, Caballero H, Marco de Lucas E, García-Porrero JA, Vázquez-Barquero A: Fiber dissection and diffusion tensor imaging tractography study of the temporoparietal fiber intersection area. Neurosurgery 72 (1 Suppl Operative):87-98, 2013

27. Martino J, De Lucas EM: Subcortical anatomy of the lateral association fascicles of the brain: a review. Clin Anat 27:563-569, 2014

28. Martino J, Vergani F, Robles SG, Duffau H: New insights into the anatomic dissection of the temporal stem with special emphasis on the inferior fronto-occipital fasciculus: implications in surgical approach to left mesiotemporal and temporoinsular structures. Neurosurgery 66 (3 Suppl Operative): $4-12,2010$

29. Meyer A: The connections of the occipital lobes and the present status of the cerebral visual affections. Trans Assoc Am Physicians 22:7-16, 1907

30. Miyamoto S, Kataoka H, Ikeda A, Takahashi J, Usui K, Takayama M, et al: A combined subtemporal and transventricular/transchoroidal fissure approach to medial temporal lesions. Neurosurgery 54:1162-1169, 2004

31. Nagata S, Rhoton AL Jr, Barry M: Microsurgical anatomy of the choroidal fissure. Surg Neurol 30:3-59, 1988

32. Niemeyer P: The transventricular amygdalohippocampectomy in temporal lobe epilepsy, in Baldwin M, Bailey P (eds): Temporal Lobe Epilepsy. Springfield, MA: CC Thomas, 1958, pp 461-482

33. Nieuwenhuys R, Voogd J, van Huijzen C: The Human Central Nervous System, ed 4. New York: Springer, 2008, p 851

34. Olivier A: Relevance of removal of limbic structures in surgery for temporal lobe epilepsy. Can J Neurol Sci 18 (4 Suppl):628-635, 1991

35. Párraga RG, Ribas GC, Welling LC, Alves RV, de Oliveira E: Microsurgical anatomy of the optic radiation and related fibers in 3-dimensional images. Neurosurgery 71 (1 Suppl Operative): $160-172,2012$

36. Rhoton AL Jr: The lateral and third ventricles. Neurosurgery 51 (4 Suppl):S207-S271, 2002

37. Rubino PA, Rhoton AL Jr, Tong X, Oliveira Ed: Three-dimensional relationships of the optic radiation. Neurosurgery 57 (4 Suppl):219-227, 2005

38. Spalding JM: Wounds of the visual pathway. Part I: The visual radiation. J Neurol Neurosurg Psychiatry 15:99-109, 1952

39. Spalding JM: Wounds of the visual pathway. Part II: The striate cortex. J Neurol Neurosurg Psychiatry 15:169-183, 1952

40. Tanriover N, Rhoton AL Jr, Kawashima M, Ulm AJ, Yasuda A: Microsurgical anatomy of the insula and the sylvian fissure. J Neurosurg 100:891-922, 2004

41. Türe U, Harput MV, Kaya AH, Baimedi P, Firat Z, Türe H, et al: The paramedian supracerebellar-transtentorial approach to the entire length of the mediobasal temporal region: an anatomical and clinical study. Laboratory investigation. J Neurosurg 116:773-791, 2012 
42. Türe U, Yaşargil DC, Al-Mefty O, Yaşargil MG: Topographic anatomy of the insular region. J Neurosurg 90:720-733, 1999

43. Türe U, Yaşargil MG, Friedman AH, Al-Mefty O: Fiber dissection technique: lateral aspect of the brain. Neurosurgery 47:417-427, 2000

44. Walsh FB, Hoyt WF: Clinical Neuro-Ophthalmology, ed 3. Baltimore: Williams \& Wilkins, 1969, pp 43-60

45. Wang F, Sun T, Li XG, Liu NJ: Diffusion tensor tractography of the temporal stem on the inferior limiting sulcus. J Neurosurg 108:775-781, 2008

46. Wen HT, Rhoton AL Jr, de Oliveira E, Cardoso AC, Tedeschi $\mathrm{H}$, Baccanelli M, et al: Microsurgical anatomy of the temporal lobe: part 1: mesial temporal lobe anatomy and its vascular relationships as applied to amygdalohippocampectomy. Neurosurgery 45:549-592, 1999

47. Wieser HG, Yaşargil MG: Selective amygdalohippocampectomy as a surgical treatment of mesiobasal limbic epilepsy. Surg Neurol 17:445-457, 1982

48. Yaşargil MG, Türe U, Yaşargil DC: Editorial. Impact of temporal lobe surgery. J Neurosurg 101:725-738, 2004

49. Yeni SN, Tanriover N, Uyanik O, Ulu MO, Ozkara C,
Karaağaç N, et al: Visual field defects in selective amygdalohippocampectomy for hippocampal sclerosis: the fate of Meyer's loop during the transsylvian approach to the temporal horn. Neurosurgery 63:507-515, 2008

\section{Author Contributions}

Conception and design: all authors. Acquisition of data: Ribas, Yagmurlu. Analysis and interpretation of data: all authors.

Drafting the article: Rhoton, Ribas, Yagmurlu. Critically revising the article: Rhoton, Ribas, Yagmurlu. Reviewed submitted version of manuscript: all authors. Approved the final version of the manuscript on behalf of all authors: Rhoton. Statistical analysis: Ribas, Yagmurlu. Administrative/technical/material support: Rhoton. Study supervision: Rhoton, Wen.

\section{Correspondence}

Albert L. Rhoton Jr., Department of Neurosurgery, University of Florida, P.O. Box 100265, Gainesville, FL 32610-0265. email: rhoton@neurosurgery.ufl.edu. 